\title{
Unmet Needs and Quality of Life: Questionnaire-Based Survey in a Follow-Up Program for Patients with Breast Cancer
}

\author{
Soysal SD ${ }^{1 *}$, Schaefer KM1, Muenst S', Mechera \\ $\mathbf{R}^{1}$, Zeindler J ${ }^{1}$, Castrezana Lopez $\mathrm{L}^{1}$, Haug $\mathbf{M}^{1}$, \\ Weber WP ${ }^{1}$ and Viehl CT $^{2}$ \\ ${ }^{1}$ University Hospital Basel, Breast Center, Switzerland \\ ${ }^{2}$ Departement of Surgery, Hospital Center Biel, \\ Switzerland \\ *Correspondling author: Soysal SD, University \\ Hospital Basel, Breast Center, Spitalstrasse 21, 4031 \\ Basel, Switzerland
}

Received: February 23, 2021; Accepted: March 13, 2021; Published: March 20, 2021

\begin{abstract}
Introduction: The objective of this self-report survey was to detect unmet needs and evaluate the quality of life of patients attending a follow-up program after breast cancer surgery.
\end{abstract}

Methods: Patients were asked to complete a standardized questionnaire consisting of 16 questions on different aspects of follow-up. The return rate of questionnaires was $84 \%$ (147 of 174 patients).

Results: All patients considered follow-up visits as useful; however, $24 \%$ of patients did not understand the basic rationale behind it. Only $38 \%$ of patients favored annual follow-up visits, while $46 \%$ demanded individual scheduling. The surgeon was the preferred follow-up specialist for $70 \%$ of patients, $59 \%$ chose the medical oncologist. Interestingly, $81 \%$ agreed that a specialized breast care nurse could coordinate and perform independent follow-up visits. Importantly, $78 \%$ of patients stated that they did not consider follow-up by phone call a valid alternative to personal follow-up, and $58 \%$ of patients reported anxiety before follow-up visits.

Discussion: The psychological burden of breast cancer follow-up seems relevant; better patient education and common decision-making may be indicated.

Keywords: Breast cancer; Follow-up program; Quality of life

\section{Abbreviations \\ ASCO: American Society of Clinical Oncology Introduction}

Breast cancer is the most frequent malignant tumor in Swiss women and worldwide, comprising $32.2 \%$ of all cancer cases [1] and accounting for $15 \%$ of all cancer-related deaths in women [2]. Mean age at diagnosis is 62 years, but the incidence of breast cancer in young and middle-aged women seems to be rising [3]. This can in part be explained by the broad implementation of screening programs and the introduction of more sensitive imaging techniques, such as digital mammography and MRI, both of which increase the detection rate of early stage breast cancer [3].

Since the 5-year survival rate of early stage breast cancer is as high as $96 \%$, the rising detection of early stage breast cancer as well as improved treatment options have led to an increasing number of women undergoing regular follow- up after their primary treatment [2].

Only scarce scientific data exists on follow-up recommendations for patients with breast cancer. Guidelines of the American Society of Clinical Oncology (ASCO) recommend physical examinations every 3 to 6 months for the first 3 years, every 6 to 12 months for years 4 and 5 and annually thereafter [4]. For women who have undergone breast- conserving surgery, a mammography should be performed 1 year after the initial mammogram and at least 6 months after completion of radiotherapy, and annually thereafter [4]. The recommendations of the Health Canada's Steering Committee on Clinical Practice Guidelines for the Care and Treatment of Breast Cancer suggest regular follow-up visits at individual frequency [5], whereas the UK guidelines recommend routine follow-up for three years only [6]. More intensive follow-up with liver ultrasound, chest radiography, biochemical profile and regular bone scans has not shown any survival benefit [7]. Therefore, routinely searching for asymptomatic metastatic disease is generally not recommended [7].

At the University Hospital of Basel Breast Center, follow-up is commonly performed by breast surgeons, radiation and medical oncologists, and consists of medical history and physical exam every 3 months for the first 3 years, every 6 months for years 4 and 5, and annually thereafter. Mammograms are obtained according to the ASCO guidelines [4].

The main goals of routine follow-up are: 1) Detection of new primaries and locoregional recurrence; 2) Diagnosis and therapy of treatment-related adverse events and complications; 3) Evaluation of quality of life including sexual well-being and of psychological sequelae, such as depression and anxiety; 4) Treatment update.

The UK recommendations to stop routine follow-up after 3 years, based on the lack of evidence of improved survival by ongoing follow-up [8], may not account for the other goals described above.
Austin J Obstet Gynecol - Volume 8 Issue 3 - 2021

Submit your Manuscript | www.austinpublishing group.com

Soysal et al. (C) All rights are reserved
Citation: Soysal SD, Schaefer KMMuenst S, Mechera R, Zeindler J, Castrezana Lopez L, et al. Unmet Needs and Quality of Life: Questionnaire-Based Survey in a Follow-Up Program for Patients with Breast Cancer. Austin J Obstet Gynecol. 2021; 8(3): 1173. 
[7]. Regular follow-up visits have the potential to reduce anxiety, long-term concerns of survivorship and psychosexual or body image related issues [9]. The psychological burden of breast cancer depends on the tumor stage and prognosis, therapy and side effects, the personality of the patient, and the availability of psychosocial support [10]. Therefore, psychosocial support must be regarded as an integral component of follow-up [10]. A survey on patients' perception of follow-up in the UK revealed that $69 \%$ did not feel comfortable raising psychological concerns for various reasons [11]. A questionnaire-based study of 79 breast cancer patients in the UK suggested that patients were not fully aware of the rationale to perform routine follow-up [7].

Due to the overall limited evidence on unmet needs of patients after surgery for breast cancer, the aim of the present study was to detect such needs and evaluate the quality of life of patients attending a standardized follow-up program. In addition, since follow-up by a specialized breast care nurse has been emphasized by several authors $[12,13]$, we evaluated preferences regarding different follow-up concepts.

\section{Patients and Methods}

\section{Patients}

During a 1-year period, 174 patients followed after breast cancer surgery at our breast Center at the University Hospital Basel were asked to complete a standardized questionnaire consisting of 16 questions on different aspects of follow-up. Questionnaires were sent out with pre-paid return envelopes. The questionnaire contained 11 closed questions with only one-answer possible and 5 questions with multiple possible answers. For closed questions, patients had the possibility to answer on an ordinal scale from 1 to 6 , with 6 being extremely satisfied, 1 being not satisfied at all, and 4 being sufficient.

Information on clinical features such as type of surgery, sociodemographic data and histopathological data (TNM stage) was obtained from our prospectively collected database (Table 1).

\section{Statistical analysis}

Data was collected in an anonymized manner and analyzed by using descriptive statistics with GraphPad (Prisme, Version 5.00).

\section{Results}

In total, 147 of 174 questionnaires were completed and returned (84\%) The majority of patients $(\mathrm{n}=76 / 52 \%)$ had $\mathrm{pT} 1$ tumors and no lymph node involvement $(\mathrm{n}=118 / 80 \%)$. Most tumors $(\mathrm{n}=107 / 73 \%)$ were invasive ductal carcinomas. The clinicopathological features of the patients are outlined in Table 1, and the detailed answers are shown in Table 2 and visualized in Figure 1.

All patients acknowledged that the follow-up visits were useful, and $11(76 \%)$ felt adequately informed on the basic rationale behind the follow-up. When asked about their satisfaction with the follow-up $(\mathrm{n}=111 / 76 \%$ of all patients reported a satisfaction of $\geq 4$, whereas 36 (24\%) of patients were not satisfied with their follow-up visits (score below 4).

Approximately two-thirds $(\mathrm{n}=101 / 69 \%)$ of patients reported examining their breasts on a monthly basis by self- palpation and inspection. When asked about their level of anxiety before the follow-
Table 1: Basic demographics of 147 patients with breast cancer undergoing follow up.

\begin{tabular}{|c|c|c|}
\hline Mean age at diagnosis (years) \pm standard & \multicolumn{2}{|c|}{$69 \pm 11.2$} \\
\hline Tumor stage & $\begin{array}{l}\text { Number } \\
\text { (n) }\end{array}$ & $\begin{array}{l}\text { Percent } \\
\text { (\%) }\end{array}$ \\
\hline pT1 & 76 & 51.7 \\
\hline рT2 & 45 & 30.6 \\
\hline рT3 & 17 & 11.6 \\
\hline pT4 & 9 & 6.1 \\
\hline \multicolumn{3}{|l|}{ Lymph node involvement } \\
\hline pNO & 118 & 80.3 \\
\hline pN1 & 23 & 15.6 \\
\hline pN2 & 6 & 4.1 \\
\hline \multicolumn{3}{|l|}{ Tumor grade } \\
\hline 1 & 39 & 26.5 \\
\hline 2 & 58 & 39.5 \\
\hline 3 & 50 & 34 \\
\hline \multicolumn{3}{|l|}{ Histologic subtype } \\
\hline Invasive ductal & 107 & 72.8 \\
\hline Invasive lobular & 17 & 11.6 \\
\hline Mucinous & 6 & 4.2 \\
\hline Apocrine & 4 & 2.8 \\
\hline Other & 13 & 8.6 \\
\hline \multicolumn{3}{|l|}{ Intrinsic subtype } \\
\hline Luminal A (ER ${ }^{+}$and/or PR ${ }^{+}$, HER2 $;$Ki-67 < 14\%) & 16 & 10.9 \\
\hline $\begin{array}{l}\text { Luminal B (HER2-negative) ER } \mathrm{ER}^{+} \text {and/or } \mathrm{PR}^{+} \text {, HER2, } \\
\qquad \mathrm{Ki}-67 \geq 14 \%)\end{array}$ & 71 & 48.3 \\
\hline Luminal B (HER2-positive) (ER ${ }^{+}$and/or $\left.\mathrm{PR}^{+}, \mathrm{HER}^{+}\right)$ & 21 & 14.3 \\
\hline HER2 type (ER- or PR', HER2+') & 12 & 8.2 \\
\hline Basal-like (ER', PR', HER2') & 27 & 18.3 \\
\hline
\end{tabular}

up visits, with 1 being not worried at all, and 6 being extremely worried, $85 / 58 \%$ stated that they felt worried $(\geq 4)$, while $62(42 \%)$ were not or only little worried. In contrast, all of the patients felt reassured after the follow-up visits, with 140 (95\%) being very or extremely reassured.

Of all patients, 56 (38\%) felt that the follow-up visits should be performed annually, while $68(46 \%)$ of patients thought that they should be scheduled on an individual basis. A majority of patients 63 (43\%) stated that the follow- up visits should continue for the rest of their lifes, whereas a minority thought that they should be stopped after 3,5 or 10 years $(17 \%, 10 \%$ and $8 \%$, respectively). Even so, 33 $(22 \%)$ of patients stated that the duration of follow-up visits should be adjusted individually.

The quality of life was reported as good (score 4 and above) by 144 (98\%) of patients, with 92 (61\%) rating their quality of life as excellent.

When asked about their sexual well-being, 111 (76\%) of patients reported that they were afraid that sexual intercourse would be painful after treatment. While $53(36 \%)$ of patients were sexually active, 75 (51\%) denied any sexual intercourse, and 19 (13\%) chose not to answer. 


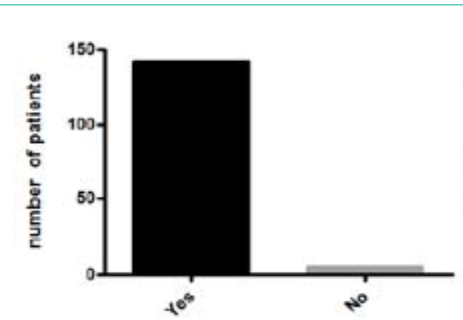

Do you consider follow up visits useful
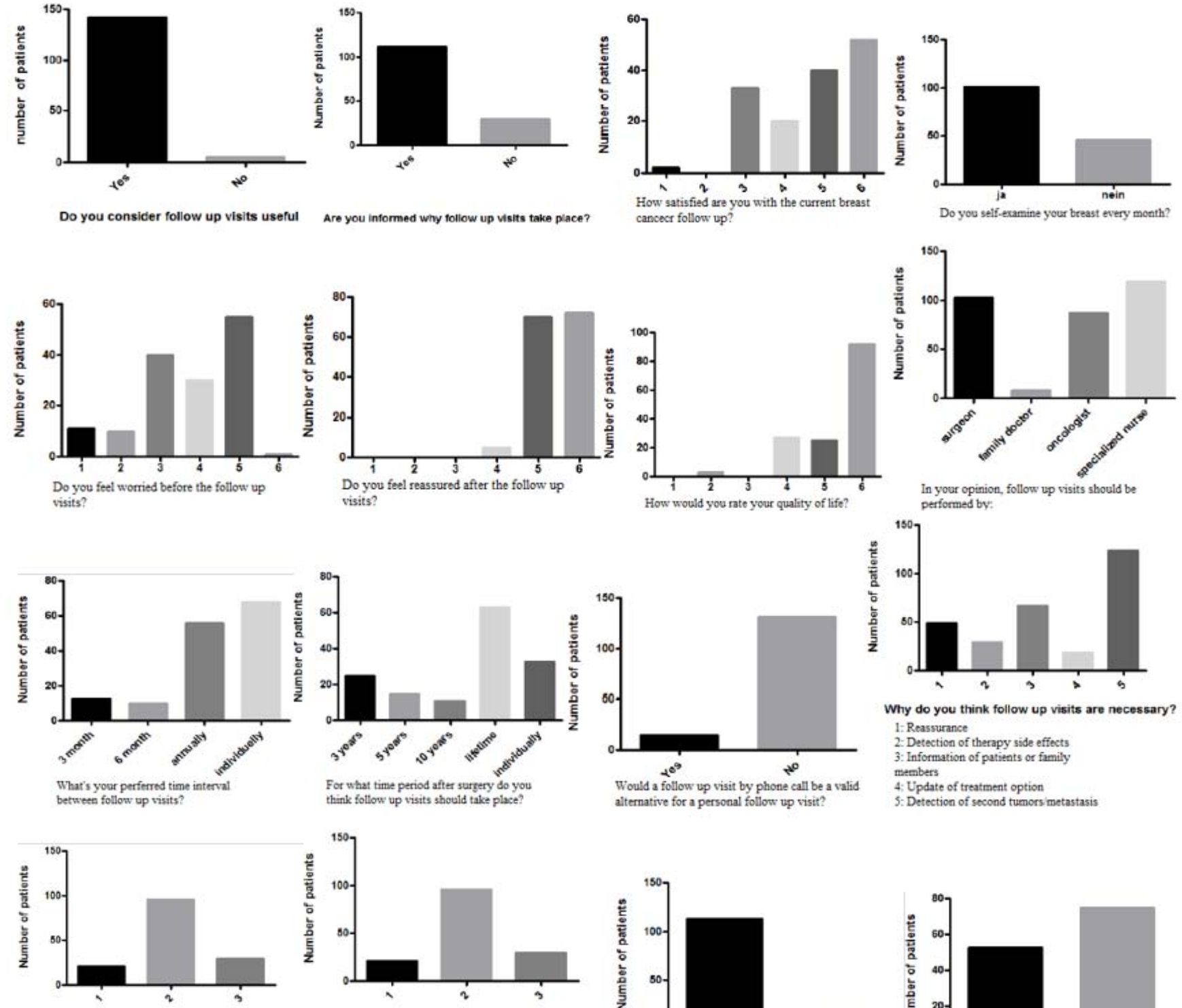

Are you currently recei
1. Psychological support!
2: Involvement of a breast

2. Involvement of a breast nurse

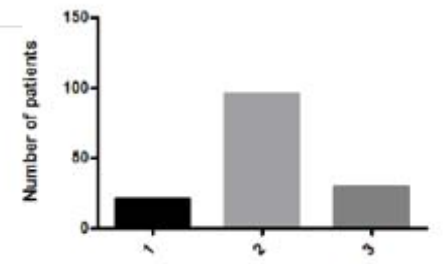

What are meaningful factors
1: Psychological support?

2: Involvement of a breast nurse

3: Informations and offers about self-belp groups?
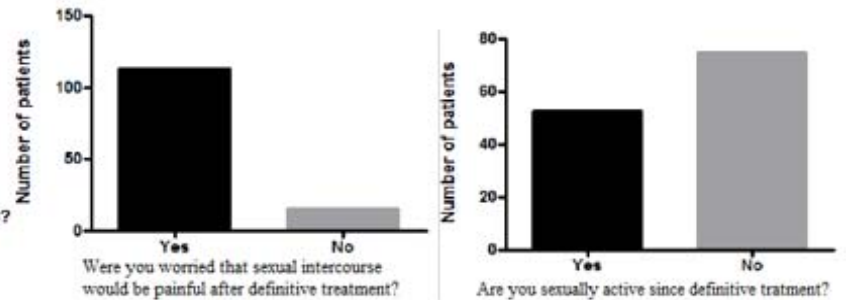

Figure 1: The clinicopathological features of the patients detailed answers.

The answer to the question who should perform the follow-up visits was surgeon in $70 \%$ of patients, while $59 \%$ chose the medical oncologist (multiple choices were possible). Interestingly, $81 \%$ of all patients agreed that a specialized breast care nurse could independently perform follow-up visits, a concept that has not yet been implemented in Switzerland. Only 5\% of patients wanted that the family practitioners perform the follow-up visits.

Most patients (78\%) denied that a follow-up by phone call would be a valid alternative to a personal follow-up exam performed by a specialized health care professional.

When asked why they thought the follow-up visits should take place (with multiple answers possible), $84 \%$ of the patients stated that their purpose should be the detection of second tumors or metastases, while $46 \%$ thought that they should be used to inform the patient as well as family members about the disease and course of treatment. One third of the patients thought that the follow-up visits should reassure them, while $20 \%$ believed that they should detect therapy side effects, and $13 \%$ expected an update on treatment options during the follow-up visits.

The majority of patients (88\%) was not seeking professional psychological care at the time of answering the questionnaire. When asked about measures that could be important for follow-up care, two-thirds (65\%) of patients stated that the follow-up visits should involve a specialized breast care nurse, while $21 \%$ wished to receive informations about support groups, and $14 \%$ requested psychological support as part of their follow-up visits. 
Table 2: Answer all patients.

\begin{tabular}{|c|c|c|c|c|c|c|c|c|c|c|c|c|}
\hline \multirow{2}{*}{ Questions } & \multicolumn{6}{|c|}{ Yes } & \multicolumn{6}{|c|}{ No } \\
\hline & \multicolumn{3}{|c|}{$\mathbf{n}$} & \multicolumn{3}{|c|}{$\%$} & \multicolumn{3}{|c|}{$\mathbf{n}$} & \multicolumn{3}{|c|}{$\%$} \\
\hline Do you consider follow up visits useful? & \multicolumn{3}{|c|}{142} & \multicolumn{3}{|c|}{96.6} & \multicolumn{3}{|c|}{5} & \multicolumn{3}{|c|}{3.4} \\
\hline Are you informed why follow up visits take place? & \multicolumn{3}{|c|}{117} & \multicolumn{3}{|c|}{79.6} & \multicolumn{3}{|c|}{30} & \multicolumn{3}{|c|}{20.4} \\
\hline Do you self-examine your breast every month? & \multicolumn{3}{|c|}{101} & \multicolumn{3}{|c|}{68.7} & \multicolumn{3}{|c|}{46} & \multicolumn{3}{|c|}{31.3} \\
\hline $\begin{array}{l}\text { Would a follow up visit by phone call be a valid alternative for a personal follow up } \\
\text { visit }\end{array}$ & \multicolumn{3}{|c|}{16} & \multicolumn{3}{|c|}{10.8} & \multicolumn{3}{|c|}{131} & \multicolumn{3}{|c|}{89.1} \\
\hline Are you currently receiving psychological care? & \multicolumn{3}{|c|}{17} & \multicolumn{3}{|c|}{11.6} & \multicolumn{3}{|c|}{130} & \multicolumn{3}{|c|}{88.4} \\
\hline $\begin{array}{l}\text { Were you worried that sexual intercourse would be painful after definitive } \\
\text { treatment? }\end{array}$ & \multicolumn{3}{|c|}{111} & \multicolumn{3}{|c|}{87.4} & \multicolumn{3}{|c|}{16} & \multicolumn{3}{|c|}{12.6} \\
\hline Are you sexually active since definitive treatment? & \multicolumn{3}{|c|}{53} & \multicolumn{3}{|c|}{41.4} & \multicolumn{3}{|c|}{75} & \multicolumn{3}{|c|}{58.6} \\
\hline Ousctions & & & & & & 3 & & 4 & & 5 & & 6 \\
\hline 8 & $\mathbf{n}$ & $\%$ & $\mathbf{n}$ & $\%$ & $\mathbf{n}$ & $\%$ & $\mathbf{n}$ & $\%$ & $\mathbf{n}$ & $\%$ & $\mathbf{n}$ & $\%$ \\
\hline Do you feel worried before the follow up visits? & 11 & 7.5 & 10 & 6.8 & 40 & 27.2 & 30 & 20.4 & 55 & 37.4 & 1 & 0.7 \\
\hline Do you feel reassured after the follow up visits? & 0 & 0 & 0 & 0 & 0 & 0 & 5 & 3.4 & 70 & 47.6 & 72 & 50 \\
\hline How would you rate your quality of life? & 0 & 0 & 3 & 2 & 0 & 0 & 27 & 18.4 & 25 & 17 & 92 & 62.6 \\
\hline
\end{tabular}

In your opinion, follow up visits should be performed by:

surgeon

family doctor

oncologist

specialized nurse

\begin{tabular}{|c|c|}
\hline $\mathbf{n}$ & $\%$ \\
\hline 103 & 32.5 \\
\hline 8 & 2.5 \\
\hline 87 & 27.5 \\
\hline 119 & 37.5 \\
\hline
\end{tabular}

\begin{tabular}{|c|c|c|}
\hline What's your preferred time interval between follow up visits? & $\mathbf{n}$ & $\%$ \\
\hline 3 months & 13 & 8.8 \\
\hline 6 months & 10 & 6.8 \\
\hline annually & 56 & 38.1 \\
\hline individually & 68 & 46.3 \\
\hline 3 years & 25 & 17 \\
\hline 5 years & 15 & 10.2 \\
\hline 10 years & 11 & 7.5 \\
\hline lifetime & 63 & 42.9 \\
\hline individually & 33 & 22.4 \\
\hline Why do you think follow up visits are necessary? & $\mathbf{n}$ & $\%$ \\
\hline reassurance & 49 & 17 \\
\hline detection of therapy side effects & 29 & 10 \\
\hline information of patient or family members & 67 & 23.3 \\
\hline update of treatment option & 19 & 6.6 \\
\hline detection of second tumors/ metastasis & 124 & 43.1 \\
\hline What are meaningful factors concerning follow up? & $\mathbf{n}$ & $\%$ \\
\hline psychological support & 21 & 14.3 \\
\hline involvement of a breast nurse & 96 & 65.3 \\
\hline informations and offers about self-help groups & 30 & 20.4 \\
\hline
\end{tabular}




\section{Discussion}

The present study is -to our knowledge- one of the first to comprehensively assess patients' perception of key elements of breast cancer follow up. Even though response bias cannot be excluded in a questionnaire-based study, the return rate of $84 \%$ and the pragmatic design of the questionnaire suggest that the results can be interpreted with confidence. While all patients considered follow-up visits as useful, $24 \%$ of the patients did not fully understand the basic rationale behind it. Hence, the need for more comprehensive patient information is obvious and has been implemented in our clinic.

In addition, the fact that $24 \%$ of patients were not satisfied with their follow-up visits is alarming, and the underlying reasons could not entirely be explored by the limitation of this questionnaire-based approach. However, the involvement of a specialized breast care nurse, support groups and structured psychological support were identified as unmet needs in this patient population.

Even though self-examination is controversial [14], 69\% of patients in this series reported to examine their breasts on a regular monthly basis. In our opinion, breast self-exams can be encouraged, since it has been reported that a total of $30-40 \%$ of potentially treatable relapses are detected by patient self-examination, and patients with ipsilateral breast relapse detected clinically do worse than those detected by self-examination or mammography $[15,16]$.

The psychological burden of routine follow-up seems relevant, with $58 \%$ of patients reporting anxiety before their follow-up visits. This percentage is comparable with a study by Paradiso et al. in which $70 \%$ of women reported feelings of anxiety before such visits [17]. Montgomery et al. stated that the benefit of follow-up might not justify its psychological burden [16]. On the upside, almost all patients (95\%) in our study felt reassured after the follow-up visit.

Importantly, only $38 \%$ of patients agreed on annual followup visits, while $46 \%$ thought that the visits should be scheduled individually depending on symptoms and personal needs. Annual visits are recommended by ASCO [4], while the Canadian Committee suggests individually scheduled follow-up visits according to individual patient's needs, and emphasize the fact that patients should be encouraged to report new persistent symptoms promptly without waiting for the next scheduled appointment [5]. Judging by our results, these later guidelines seem to be more consistent with patient's conceptions. A considerable percentage of patients (22\%) stated that the duration of follow-up visits should be adapted individually.

Potentially treatable relapse occurs at a constant rate of $1-1.5 \%$ per year for at least 10 years, the majority of relapses occurring after 3 years of follow-up [16]. Since patients with late relapses tend to do particularly well, every effort should be undertaken to diagnose these late relapses at an early stage, and thus it is our belief that the offered follow-up should not stop at 3 years [16], as it has been suggested by the UK guidelines [8]. We support the ASCO recommendations, which suggest 6-monthly clinical visits for the first 5 years and annually thereafter [4].

A majority of patients (76\%) reported that they were afraid that sexual intercourse would be painful after treatment for breast cancer, and only $36 \%$ were sexually active. These findings confirm previous reports of significant deterioration of sexual well-being after diagnosis and treatment for breast cancer [18]. Health professionals involved in follow-up visits play an important role in alleviating concerns surrounding sexual well-being after breast cancer, and it is thus important to address the issue of sexual function during followup visits $[19,20]$.

The prediction of a $48 \%$ increased need for cancer services by 2020 forecasts a parallel increase in workload for clinics performing oncological follow-up [21]. In this study performed on a breast surgery service, $70 \%$ of patients preferred follow-up by a surgeon, and $59 \%$ by a medical oncologist. Importantly, $81 \%$ of patients agreed that a specialized breast care nurse could coordinate and perform independent follow-up visits, a concept that has not yet been widely implemented in Switzerland. Similar results were reported in a study by Kwast et al. [22]. Patients stated that breast care nurses were easily accessible, had more time for the individual patient and were perceived more socially empathic [22]. Koinberg et al. who investigated nurse led follow up on demand versus physician (surgeon or oncologist) follow-up after breast cancer treatment reported no difference in anxiety and depression as well as patient satisfaction between the two groups [23]. Furthermore, there was no difference between the groups regarding time to recurrence of disease or death [23]. These results are confirmed by Kimman et al. who demonstrated that overall patient satisfaction was similar if patients were followed by a physician alternating with a breast care nurse compared with follow-up by a physician alone [13]. In the trial by Baildam et al. the Fallowfield Satisfaction with Consultation Questionnaire revealed that women were significantly more satisfied with their consultation with a nurse than those seen by a doctor $(\mathrm{P}<0.001)$ [24].

The role of specialized breast care nurses has also been discussed at the 2013 St Gallen consensus conference, where the panelists agreed that regular follow-up supervised by a nurse specialist in person or by telephone would be acceptable for surveillance [25]. While in our survey, patients agreed with a more important role of the breast care nurse during personal follow-up, $78 \%$ of patients stated that they did not consider follow-up by phone call a valid alternative. This is contrary to results from a recent Australian study encompassing 722 breast cancer patients suggesting that telephone interviews could play an important role [12]. Similarly, a study from the UK compared traditional outpatient clinics follow-up with phone based follow-up by breast care nurses and reported that patients with telephone follow-up had higher levels of satisfaction but not higher levels of anxiety. No difference between the two groups was found regarding detection of recurrent disease [26]. Therefore, even though telephone follow-up is not widely accepted by patients and health care providers in Switzerland, some authors suggest that it could be a valid alternative to clinical follow-up visits [26].

Most patients (84\%) felt that the main purpose of clinical followup was to detect local or distant relapses of disease. Even though this certainly is a key element, a study by Montgomery et al showed that the majority of relapses in a cohort of 198 breast cancer patients was not detected by physical exam, and clinicians must be aware of these limitations [27]. Almost half of the patient's (46\%) felt that these visits should be used to inform patients and their families about the disease 
and treatment course, an aspect that is frequently underestimated by the treating physician. Only $20 \%$ of the patients thought that the follow-up visits were performed in order to detect therapy-related side effects. This is important, since a study by Schmitz et al. showed that at 6 years after diagnosis, more than $60 \%$ of breast cancer patients were experiencing one or more adverse treatment effects that could be influenced by targeted intervention [28].

Although psychological syndromes such as depression and anxiety are common among cancer patients [29], the majority of patients (88\%) in our study population were not receiving psychological care. One potential explanation is that these needs are not detected and sufficiently addressed during follow up, and psychological support was not offered. This is supported by the fact that $21 \%$ of patients would like to receive information about support groups and $14 \%$ requested psychological support as part of their follow-up visits. In many contemporary breast centers, a psycho-oncological unit provides specialized care and could be used to meet those needs in addition to the important contribution of breast care nurses.

The present study at a University Hospital in Switzerland shows that current standards in breast cancer follow-up do not fully meet patient needs, and that not all patients are aware of the rationale behind routine follow-up. As a consequence of this study, a more significant role has been assigned to a specialized breast care nurse at our institution, who coordinates the multidisciplinary approach and remains the reference person for our patients. Additionally, patients are informed more comprehensively and special attention is given to their requests.

\section{Statement of Ethics}

The study protocol was approved by the local Ethics Committee (Ethikkommission beider Basel, EKBB, Number/ID of the approval(s): $322 / 10$ ) and all patients gave written informed consent.

\section{Author Contribution}

SD Soysal and KM Schaefer contributed equally to this study. WP Weber and CT Viehl contributed equally to this study.

\section{References}

1. Krebs in der Schweiz: wichtige Zahlen. Krebsliga Schweiz. 2020.

2. Jemal A, Siegel R, Ward E, Hao Y, Xu J, Thun MJ. Cancer statistics 2009. CA Cancer J Clin. 2009; 59: 225-249.

3. Levi F, Te VC, Maspoli M, Randimbison L, Bulliard JL, La Vecchia C. Trends in breast cancer incidence among women under the age of forty. $\mathrm{Br} \mathrm{J}$ Cancer. 2007; 97: 1013-1014

4. Khatcheressian JL, Hurley P, Bantug E, Esserman LJ, Grunfeld E, Halberg $\mathrm{F}$, et al. Breast cancer follow-up and management after primary treatment: American Society of Clinical Oncology clinical practice guideline update. J Clin Oncol. 2013; 31: 961-965.

5. Grunfeld E, Dhesy-Thind S, Levine M. Clinical practice guidelines for the care and treatment of breast cancer: follow-up after treatment for breast cancer (summary of the 2005 update). CMAJ. 2005; 172: 1319-1320.

6. Anderson BO, Carlson RW. Guidelines for improving breast health care in limited resource countries: the Breast Health Global Initiative. J Natl Compr Canc Netw. 2007; 5: 349-356

7. Dixon JM, Montgomery DA. Extended follow-up of breast cancer patients in clinic wastes time for both patients and doctors: the case for. Breast Cancer Res. 2008; 10: S7.
8. Guidance on Cancer Services Improving Outcomes in Breast Cancer. 2002.

9. Sheppard C. Breast cancer follow-up: literature review and discussion. Eur J Oncol Nurs. 2007; 11: 340-347.

10. Rauchfuss MBJ, Neises M, Danzer D, Klatt BF. Psychoonkologische Versorgung in einem Brustzentrum. Geburtsh Frauenheilk. 2004; 64: 11581166.

11. Pennery E, Mallet J. A preliminary study of patients' perceptions of routine follow-up after treatment for breast cancer. Eur J Oncol Nurs. 2000; 4: 138145; discussion 146-147.

12. Bessen T, Chen G, Street J, Eliott J, Karnon J, Keefe D, et al. What sort of follow-up services would Australian breast cancer survivors prefer if we could no longer offer long-term specialist-based care? A discrete choice experiment. Br J Cancer. 2014; 110: 859-867.

13. Kimman ML, Dellaert BG, Boersma LJ, Lambin P, Dirksen CD. Follow-up after treatment for breast cancer: one strategy fits all? An investigation of patient preferences using a discrete choice experiment. Acta Oncol. 2010; 49: 328-337.

14. Kettritz U. Screening of Breast Cancer-an Eternal Discussion Revisited. Breast Care (Basel). 2010; 5: 119-120.

15. Montgomery DA, Krupa K, Jack WJL, Kerr GR, Kunkler IH, Thomas J, et al. Changing pattern of the detection of locoregional relapse in breast cancer: the Edinburgh experience. Br J Cancer. 2007; 96: 1802-1807.

16. Montgomery DA, Krupa K, Cooke TG. Follow-up in breast cancer: does routine clinical examination improve outcome? A systematic review of the literature. Br J Cancer. 2007; 97: 1632-1641.

17. Paradiso A, Nitti P, Frezza P, Scorpiglione N. A survey in Puglia: the attitudes and opinions of specialists, general physicians and patients on follow-up practice. G.S.Bio.Ca.M. Ann Oncol. 1995; 6: 53-56

18. Barsotti Santos D, Ford NJ, dos Santos MA, Vieira EM. Breast cancer and sexuality: the impacts of breast cancer treatment on the sex lives of women in Brazil. Cult Health Sex. 2014.

19. Schultz WC, HB Van De Wiel. Sexuality, intimacy, and gynecological cancer. J Sex Marital Ther. 2003; 29: 121-128.

20. Ussher JM, J Perz, Gilbert E. Changes to sexual well-being and intimacy after breast cancer. Cancer Nurs. 2012; 35: 456-465.

21. Erikson C, Salsberg E, Forte G, Bruinooge S, Goldstein M. Future supply and demand for oncologists: challenges to assuring access to oncology services. J Oncol Pract. 2007; 3: 79-86.

22. Kwast $A B$, Drossaert $C H$, Siesling S. Breast cancer follow-up: from the perspective of health professionals and patients. Eur J Cancer Care (Engl). 2013; 22: 754-764.

23. Koinberg IL, Fridlund B, Engholm GB, Holmberg L. Nurse-led follow-up on demand or by a physician after breast cancer surgery: a randomised study. Eur J Oncol Nurs. 2004; 8: 109-117; discussion 118-120.

24. Baildam AD, Thompson L, Bundred N, Hopwood P. Nurse-led surgical follow up clinics for women treated for breast cancer-a randomised controlled trial. Breast Cancer Research and Treatment. 2004; 88: S136-S137.

25. Harbeck N, C Thomssen, Gnant M. St. Gallen 2013: brief preliminary summary of the consensus discussion. Breast Care (Basel). 2013; 8: 102109.

26. Beaver K, Tysver-Robinson D, Campbell M, Twomey M, Williamson S, Hindley A, et al. Comparing hospital and telephone follow-up after treatment for breast cancer: randomized equivalence trial. BMJ. 2009; 338: a3147.

27. Montgomery DA, K Krupa, Cooke TG. Locoregional relapse after breast cancer: most relapses occur late and are not clinically detected. Breast J. 2009; 15: 163-167.

28. Schmitz KH, Speck RM, Rye SA, DiSipio T, Hayes SC. Prevalence of breast cancer treatment sequelae over 6 years of follow-up: the Pulling through Study. Cancer. 2012; 118: 2217-2225. 
29. Weinberger T, Forrester A, Markov D, Chism K, Kunkel EJ. Women at a dangerous intersection: diagnosis and treatment of depression and related disorders in patients with breast cancer. Psychiatr Clin North Am. 2010; 33 409-422. 